\title{
Granulomatous amebic encephalitis caused by Acanthamoeba in an immuncompetent child
}

\author{
Murat Sütçü ${ }^{1}$, Hacer Aktürk ${ }^{1}$, Sezen Gülümser-Şişko², Manolya Acar ${ }^{1}$, Oğuz Bülent Erol ${ }^{3}$, \\ Ayper Somer ${ }^{1}$, Bilge Bilgiç4, Nuran Salman ${ }^{4}$ \\ ${ }^{1}$ Division of Infectious Diseases, Departments of ${ }^{2}$ Pediatrics, ${ }^{3}$ Radiology and ${ }^{4}$ Pathology, Istanbul University Istanbul \\ Faculty of Medicine, Istanbul, Turkey. \\ E-mail: sutcu13@yahoo.com \\ Received: 24th May 2017, Revised: 8th July 2017, Accepted: 23th July 2017
}

SUMMARY: Sütçü M, Aktürk H, Gülümser-Şişko S, Acar M, Erol OB, Somer A, Bilgiç B, Salman N. Granulomatous amebic encephalitis caused by Acanthamoeba in an immuncompetent child. Turk J Pediatr 2018; 60: 340-343.

Acanthamoeba may lead to granulomatous amebic encephalitis (GAE) with high mortality rates generally in patients with immunosupression and/or chronic disease. Here, we present a rare GAE case, who was a previously healthy child. A Georgian 9 year old boy presented with focal seizure on his left arm and confusion. Since computed tomography (CT) demonstrated hypodense lesion on right occipital lobe, brain biopsy was performed. Histopathological examination of the biopsy material revealed Acanthamoeba cysts and trophozoites together with granulomatous inflammation. The patient, who had no clinical and laboratory findings consistent with immunedeficiency, was diagnosed as GAE. He was treated with a combination drug therapy. Even if it is very rare, amebic meningoencephalitis may also be seen in immunocompetent children, as in our case. Definitive diagnosis is made by microbiological and histopathological examination of brain biopsy material.

Key words: Acanthamoeba, child, granulomatous amebic encephalitis, immuncompetent.

Naegleria (Naegleria fowleri), Acanthamoeba (Acanthamoeba castellanii ve Acanthamoeba culbertsoni), Sappiniadiploidea and Balamuthia (Balamuthia mandrillaris) are free-living amoebea that can cause infections in humans. ${ }^{1}$ Acanthamoeba spp are widely distrubuted in the environment and have been isolated from soil, air, seawater, domestic tap water, bottled water, and even dust in the air. They can cause granulomatous amebic encephalitis (GAE) mostly in patients with underlying immune deficiency or chronic disorders together with amebic keratitisin contact lens users.

Granulomatous amebic encephalitis is an insidious onset chronic disease with high mortality. It is caused by the amoeba entering the body through an open wound. Trophozoites then spread to the brain by hematogenous route. Acanthamoeba cysts suspended in air can also reach alveoli. ${ }^{2,3}$ GAE is characterized by neurological symptoms including headache, convulsions, somnolence and unilateral paralysis in some cases. Herein, we report a previously healthy boy with GAE treated with antimicrobial therapy.

\section{Case Report}

A nine-year-old previously healthy Georgian boy presented to the emergency department with the complaint of loss of conciousness and focal clonic seizure localized on his left arm. Physical examination revealed no abnormality and somatic growth was appropriate for his age. Complete blood count was normal (white blood cell count $8000 / \mu$ l, neutrophil $27 \%$, lymphocyte $55 \%$, hemoglobin level $11.8 \mathrm{~g} / \mathrm{dl}$, platelet count 456000/ $\mu \mathrm{l}$ ). Routine biochemistry revealed no abnormality. Computerized cranial tomography showed mass lesion on right occipital lobe. Hyperintense lesion $3 \times 3 \mathrm{~cm}$ in diameter with perilesional edema was also observed in craniospinal magnetic resonance

This case report was presented at the 33rd Annual Meeting of the European Society for Paediatric Infectious Diseases (ESPID). 
image (Fig. 1). Lumbar puncture had not been performed because of herniation risk. Diagnostic biopsy was performed by stereotactic surgery. Biopsy material revealed central coagulation necrosis in brain tissue together with intense granulomatous inflamation (Fig. 2). Acanthamoeba cyst formation together with trophozoites that were weakly stained with periodic acid-schiff (PAS) within macrophages were observed (Fig. 3). Cerebrospinal fluid (CSF) obtained during surgery was purulent and revealed lymphocytes on microscopic examination. CSF was cultured for parasitic investigation but did not yield any growth. He was diagnosed with granulomatous

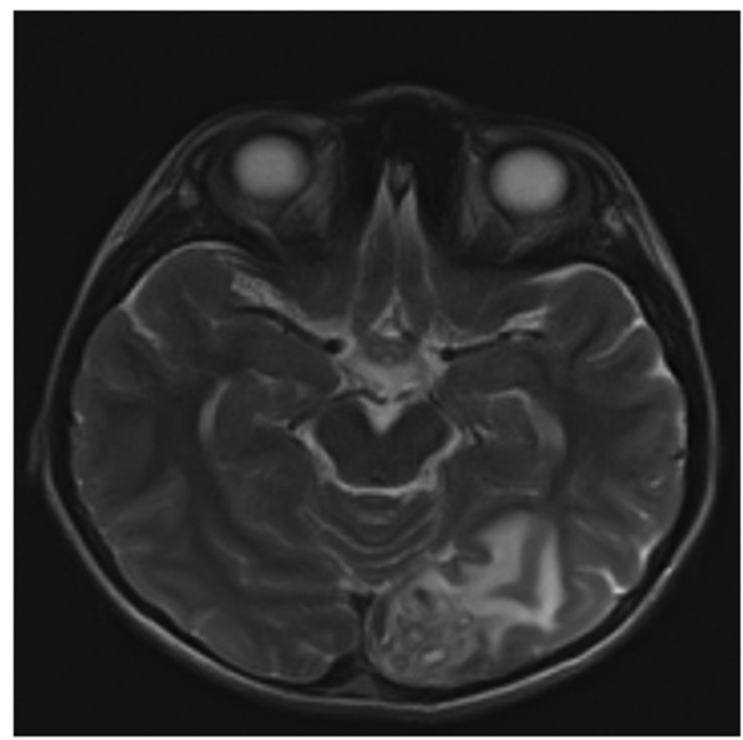

Fig. 1. Cranial MRI, hyperintense $3 \times 3 \mathrm{~cm}$ mass lesion with cystic components and perilesional edema on right parietal lobe.

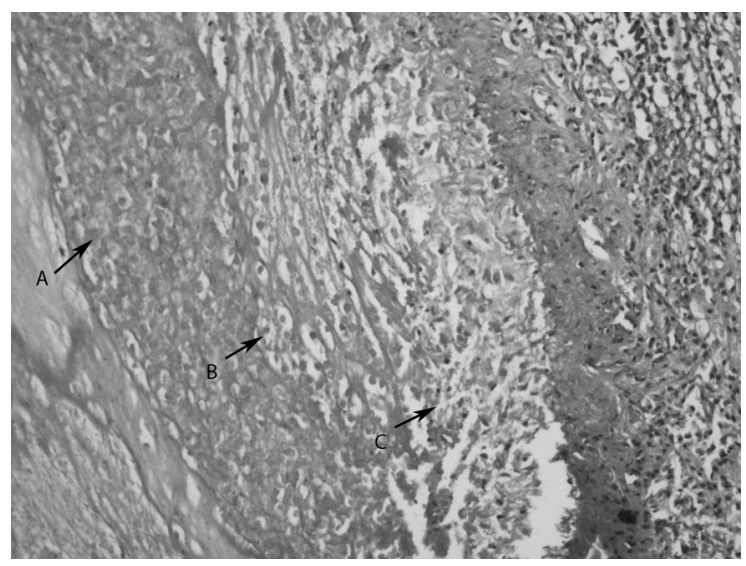

Fig. 2. Brain tissue with intense granulomatous inflammation (A), histiyocytes (B) and central coagulation necrosis (C). (hematoxylin-eosin stain; x200). amebic encephalitis with present findings. His history revealed that he often swam in lake water and had a head trauma a year ago. When he was evaluated for possible immune deficiency, absolute lymphocyte count, serum immunoglobulin levels, lymphocyte subgroup analysis were all in normal range. (IgG:893 (620-1300) $\mathrm{mg} / \mathrm{dl}$, IgA:104 (30-230) $\mathrm{mg} / \mathrm{dl}$, IgM:94 (52-240) mg/dl CD3 $+\mathrm{T}$ cell: \% 63, CD4+ T helper cell: \%35, CD8 + T cytotoxic cell:\%24, CD19+ B cell:\%15, CD16+56+ natural killer cell:\%18). Nitroblue tetrazolium test (NBT) for chronic granulomatous disease was also normal. Serologic tests for HIV, cytomegalovirus IgM, toxoplasma IgM were negative. Thorax computerized tomography and abdominal ultrasonography performed for disseminated amebic infection were normal. He was started on fluconazole, rifampisin and trimethoprim sulfamethoxazole treatment. Surgical intervention could not be performed since the mass was big, deep seated and close to visual center. On the third month of therapy, he was asymptomatic and cranial MRI showed no difference in the size of mass lesion. His therapy was tapered as liposomal amphoterisin $\mathrm{B}$, primetamin, rifampisin and trimethoprim sulfamethoxazole combination treatment and in order to evaluate for possible surgery radiologic examination was planned for one month later. But because of socioeconomic factors parents decided to go to their own country and the patient was lost to follow up. Written informed consent has been obtained from the parents of the patient.

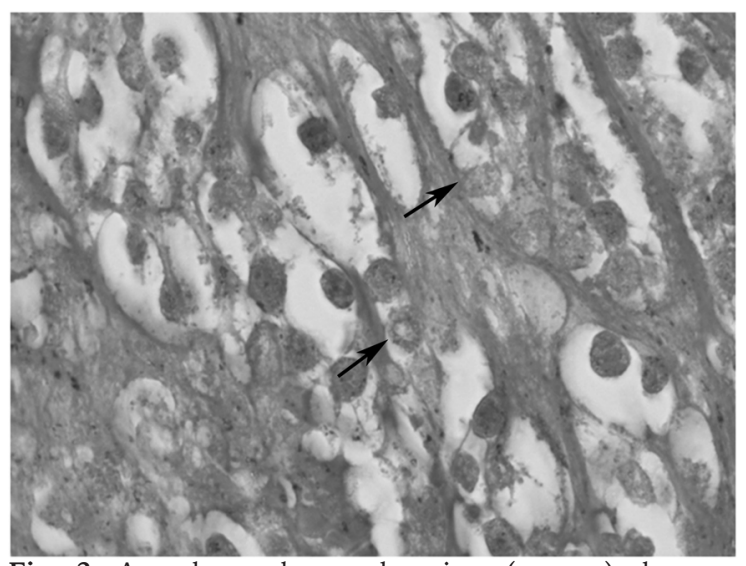

Fig. 3. Acanthomeoba trophozoites (arrows) that are weakly stained with periodic acid-schiff (PAS) within macrophages (x1000) 


\section{Discussion}

Acanthamoeba related amebic central nervous system (CNS) infections are generally named as granulomatous amebic encephalitis because of histopathologic appeareance. GAE is a higly mortal opportunistic disease that is mostly encountered in patients with immune deficiency or chronic disorder. ${ }^{4}$ Organ transplant recipients, HIV infection, hematologic malignancy, long term corticosteroid therapy, diabetes mellitus and chronic liver failure are main risk factors for GAE and disseminated amibic disease. ${ }^{2,5,6}$ In the current literature; immunecompetent cases like ours, are rarely presented with GAE. ${ }^{7,8}$ One of them is an adult patient reported from our country. ${ }^{9}$ Our case possesses significant importance since he is the first pediatric case from our country.

Acanthamoeba trophozoites enter the human body through the lungs, skin, paranasal sinuses and spread hematogenously to the CNS. ${ }^{2}$ Initial infection must have been undiagnosed in our case. Whether GAE was related with possible CSF leak secondary to head trauma and lake water swimming habit is uncertain. Hence no pathologic evidence supporting this hypothesis is appearent. Clinical findings of GAE are subtle and show great variability. As well as focal neurologic symptoms like hemiparesis, cranial nerve palsy, diplopia and cerebellar ataxia can be initial findings, non specific presentations like headache, mental status change, lethargy, convulsions, meningeal irritation signs can be observed. Intermittent subfebrile fever and vomiting can accompany. Incubation period is reported between several weeks to months. ${ }^{2}$

Diagnosis of GAE can be made either by the histopathologic examination or the isolation of the pathogen from brain biopsy material. CSF findings and radiologic investigations are non specific. Cranial imaging may show abcess, hemorrhagic involvement or tumor like mass lesion. These lesions are usually multifocal and can be observed in cortex, brain stem or spinal cord. ${ }^{10}$ Increased CSF pressure, lymphocyte dominant pleocytosis, slightly elevated protein level with mild decrease in glucose are common. Most of the time CSF is sterile and direct microbiologic investigation show no abnormality. Nevertheless, presence of trophozoites or cultural growth in pediatric cases have been reported. ${ }^{2,11}$ In our case, mass lesion was observed in cranial MRI, but lumbar puncture could not be performed because of herniation risk. Although CSF obtained during surgery was purulent, microbiologic culture did not yield any growth. Histopathologic examination of brain biopsy material showed acanthamoeba trophozoites.

Because of insufficient immune response, delay in diagnosis or ineffective antimicrobial therapy, GAE can rarely be treated effectively. Although there is no standard therapy regimen, the most successful treatment is reported as combination of pentamidin, sulphadiazine, flucytosine and fluconazole together with surgical exicion. ${ }^{2,7}$ Liposomal amphotericin B, voriconazole and miltefosine are alternative treatment options. Treatment success has been increased in patients after sugery. ${ }^{12,13}$ In our patient fluconazole, rifampisin and trimethoprim sulfamethoxazole combination was started initially because of the reported data regarding efficacy of this regimen in immune competent patients and availability of drugs. But, surgical intervention could not be performed since the size and location of the mass was inconvenient. In addition, there was no progress in the size of mass under antimicrobial therapy and the patient was asymptomatic. Surgery was planned in the case of progression or the development of neurological finding. But the patient's follow up could not be achieved since he went to his own country.

In conclusion, like our case, GAE can also be seen in an immunecompetent hosts without predisposing factors. It should be considered in differential diagnosis of patients with prolonged and nonspesific CNS symptoms. Examination of brain tissue sample with biopsy is crucial for diagnosis.

\section{REFERENCES}

1. Visvesvara GS, Moura H, Schuster FL. Pathogenic and opportunistic free-livingamoebae: Acanthamoeba spp., Balamuthia mandrillaris, Naegleria fowleri, and Sappinia diploidea. FEMS Immunol Med Microbiol 2007; 50 : $1-26$.

2. McNeil C, Singh U. Acanthamoeba species. In: Long SS, Pickering LK, Prober GC, eds. Principles and Practice of Pediatric Infectious Diseases, fourth ed. Philadelphia: Churchill Livingstone, 2012: 1295-1298.

3. Stockman LJ, Wright CJ, Visvesvara GS, Fields BS, Beach MJ. Prevalence of Acanthamoeba spp. and other free-living amoebae in household water, Ohio, USA- 
1990-1992. Parasitol Res 2011; 108: 621-627.

4. Ferrante A.Free-living amoebae: Pathogenicity and immunity. Parasite Immunol 1991; 13: 31-47.

5. Steinberg JP, Galindo RL, Kraus ES, Ghanem KG. Disseminated acanthamebiasis in a renal transplant recipient with osteomyelitis and cutaneous lesions: Case report and literature review. Clin Infect Dis 2002; 35: e43-e49.

6. Sison J, Kemper CA, Loveless M, McShane D, Visvesvara GS, Deresinski SC. Disseminated acanthamoeba infection in patients with AIDS: Case reports and review. Clin Infect Dis 1995; 20: 1207-1216.

7. Zamora A, Henderson H, Swiatlo E. Acanthamoeba encephalitis: A case report and review of therapy. Surg Neurol Int 2014; 5: 68

8. Binesh F, Karimi M, Navabii H. Unexpected postmortem diagnosis of acanthamoeba meningoencephalitis in an immunocompetent child. BMJ Case Rep 2011; 2011. pii: bcr0320113954.

9. Sarica FB, Tufan K, Cekinmez M, Erdoğan B, Altinörs $\mathrm{MN}$. A rare but fatal case of granulomatous amebic encephalitis with brain abscess: The first case reported from Turkey. Turk Neurosurg 2009; 19: 256-259.

10. Marciano-Cabral F, Cabral G. Acanthamoeba spp. as agents of disease in humans. Clin Microbiol Rev 2003; 16: 273-307.

11. Saxena A, Mittal S, Burman P, Garg P. Acanthameba meningitis with successful outcome. Indian J Pediatr 2009; 76: 1063-1064.

12. Singhal T, Bajpai A, Kalra V, et al. Successful treatment of Acanthamoeba meningitis with combination oral antimicrobials. Pediatr Infect Dis J 2001; 20: 623-627.

13. Webster D, Umar I, Kolyvas G, et al. Case Report Treatment of granulomatous amoebic encephalitis with voriconazole and miltefosine in an immunocompetent soldier. Am J Trop Med Hyg 2012; 87: 715-718. 